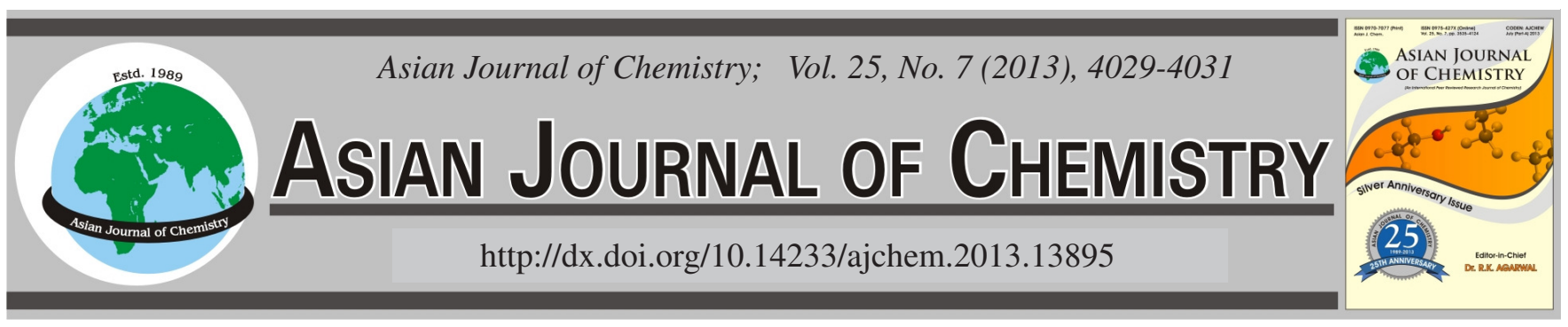

\title{
Optimization and Antifungal Activity of Amide Analogues
}

Hui Feng ${ }^{1}$, Li Leng ${ }^{2}$, Jia Liu ${ }^{1}$, Yuanmou Tang ${ }^{1}$, Pengcheng Tang ${ }^{1}$, Chixiang Zhang ${ }^{1}$, Xioarong Tang $^{1}$ and Shirong Jiao ${ }^{1, *}$

${ }^{1}$ School of Bioengineering, Xihua University, Chengdu 610039, P.R. China

${ }^{2}$ School of Life Science, Sichuan University, Chengdu 610064, P.R. China

*Corresponding author: Tel: +86 13882002899; E-mail: huif2010@163.com

(Received: 12 April 2012;

Accepted: 23 January 2013)

AJC-12746

Using salicylic acid as a lead compound, a series of its analogues (compounds 1-16) were designed and synthesized. Their activity of anti-
pathogenic fungi of plants has been evaluated in the laboratory. The results showed that these compounds had certain antifungal activity
against Sclerotinia sclerotiorum and Bipolaris maydis (Nisikado et Miyake) Shoem. Among them, the inhibition of growth for $2-(3-$
fluorophenylcarbamoyl)phenyl acetate (1) and 2-(3-chlorophenylcarbamoyl)phenyl acetate (2) reached $91.1 \%, 92.8 \%$ and $90.1 \%, 90.1$
$\%$ at a concentration of $100 \mathrm{mg} \mathrm{L}^{-1}$, respectively.
Key Words: Antifungal activity, Amide analogues, Synthesis.

\section{INTRODUCTION}

In agriculture, crop disease is the major issues in agricultural production. Crop yields cutting down more than $10 \%$ are caused by them. Meanwhile, fungal diseases are about $80 \%$ in the plant disease $\mathrm{e}^{1,2}$. Many kinds of fungicides are chemical fungicides. However, their over use has brought about side effects, such as the contamination of the environment and occurrence of resistance ${ }^{3}$. Botanical fungicides play an important role in controlling pathogenic fungi of plants ${ }^{4-6}$. Nevertheless, they have obvious drawbacks such as low yield, instability, complex components and limited antifungal spectrum. Salicylic acid (Fig. 1) is a natural compound existing in many plants. It can help plants to disease resistance ${ }^{7-18}$. Thus, salicylic acid used as a lead structure to synthesize new fungicides is meaningful.<smiles>O=C(O)c1ccccc1O</smiles>

Fig. 1. Chemical structure of salicylic acid

\section{EXPERIMENTAL}

Sclerotinia sclerotiorum and Bipolaris maydis (Nisikado et Miyake) Shoem were obtained from the Chinese Academy of Agricultural Sciences. They were preserved at $4{ }^{\circ} \mathrm{C}$. All chemicals and solvents were purchased from commercial sources unless specified otherwise. IR spectra were recorded on a Thermofisher Nicolet-6700 spectrophotometer. ${ }^{1} \mathrm{H}$ NMR spectra were taken on a Varian Unity Inova-400 instrument using deuteron chloro-form as the solvent.

Synthesis of target compounds: The target compounds were synthesized according to the route shown in Fig. 2 and their yield was not optimized. Benzenamine or its derivatives (30 mmol) and pyridine (30 mmol) were dissolved in $\mathrm{CH}_{2} \mathrm{Cl}_{2}$ $(10 \mathrm{~mL})$. The mixture was stirred and heated to $30{ }^{\circ} \mathrm{C}$. 2 (Chlorocarbonyl)phenyl acetate or 2-methoxybenzoyl chloride $(30 \mathrm{mmol})$ were dissolved in $\mathrm{CH}_{2} \mathrm{Cl}_{2}(10 \mathrm{~mL})$ and it was slowly mix under stirring 3-5 h until the reaction was complete. The precipitate was filtered and washed with distilled water. The pure compounds 1-16 were obtained by re-crystallization in anhydrous ethanol.

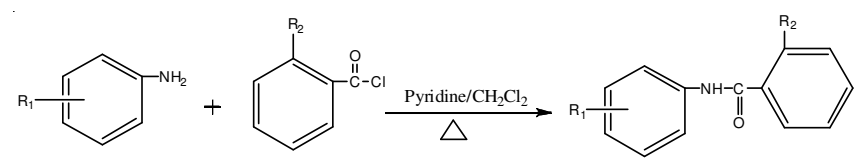

Fig. 2. Synthesis of target compounds 1-16

Compound 1: White crystal, yield: $20 \%$, m.p. $116-117^{\circ} \mathrm{C}$; IR $\left(\mathrm{KBr}, v_{\max }, \mathrm{cm}^{-1}\right): 3296,3261,3187,3077,1770,1743,1666$, 1593, 1537, 1484, 1450, 1368, 1316, 1227, 1162, 1135, 785, 775, 751, 696, 680, 583; ${ }^{1} \mathrm{H}$ NMR (400 MHz, $\left.\mathrm{CDCl}_{3}\right) \delta(\mathrm{ppm})$ : 2.32(s, 3H), $7.13(\mathrm{t}, J=8.4 \mathrm{~Hz}, 2 \mathrm{H}), 7.26(\mathrm{t}, J=8.0 \mathrm{~Hz}, 1 \mathrm{H}), 7.33$ $(\mathrm{t}, J=8.0 \mathrm{~Hz}, 1 \mathrm{H}), 7.40(\mathrm{~d}, J=8.0 \mathrm{~Hz}, 1 \mathrm{H}), 7.51(\mathrm{t}, J=7.2 \mathrm{~Hz}$, 1H), $7.74(\mathrm{~s},, 1 \mathrm{H}), 7.79$ (d, $J=8.0 \mathrm{~Hz}, 1 \mathrm{H}), 8.14$ (s, 1H). 
Compound 2: White crystal, yield: $27 \%$, m.p. 126$127^{\circ} \mathrm{C}$; IR (KBr, $\left.v_{\max }, \mathrm{cm}^{-1}\right): 3296,3261,3187,3115,3077$, 1770, 1743, 1676, 1666, 1593, 1537, 1484, 1450, 1368, 1316, 1203, 1162, 1135, 785, 775, 751, 696, 583; ${ }^{1} \mathrm{H}$ NMR (400 $\left.\mathrm{MHz}, \mathrm{CDCl}_{3}\right) \delta$ (ppm): 2.31 (s, 3H), $7.12(\mathrm{t}, J=8.0 \mathrm{~Hz}, 2 \mathrm{H})$, 7.23-7.27 (m, 1H), $7.31(\mathrm{t}, J=8.0 \mathrm{~Hz}, 1 \mathrm{H}), 7.39$ (d, $J=8.0$ $\mathrm{Hz}, 1 \mathrm{H}), 7.49$ (t, $J=8.0 \mathrm{~Hz}, 2 \mathrm{H}), 7.76(\mathrm{t}, J=8.0 \mathrm{~Hz}, 1 \mathrm{H}), 8.18$ (s, 1H).

Compound 3: White crystal, yield: $30 \%$, m.p. 119$120^{\circ} \mathrm{C}$; IR $\left(\mathrm{KBr}, v_{\max }, \mathrm{cm}^{-1}\right): 3293,3258,3118,3076,1768$, 1742, 1666, 1609, 1589, 1480, 1449, 1367, 1318, 1192, 1134, 1095, 783, 774, 751, 703, 679, 583; ${ }^{1} \mathrm{H}$ NMR $(400 \mathrm{MHz}$, $\left.\mathrm{CDCl}_{3}\right) \delta(\mathrm{ppm}): 2.31(\mathrm{~s}, 3 \mathrm{H}), 7.13(\mathrm{~d}, J=8.0 \mathrm{~Hz}, 1 \mathrm{H}), 7.19(\mathrm{t}$, $J=8.0 \mathrm{~Hz}, 1 \mathrm{H}), 7.26(\mathrm{t}, J=6.4 \mathrm{~Hz}, 1 \mathrm{H}), 7.31(\mathrm{t}, J=8.0 \mathrm{~Hz}$, $1 \mathrm{H}), 7.45-7.52(\mathrm{~m}, 2 \mathrm{H}), 7.77(\mathrm{~d}, J=8.0 \mathrm{~Hz}, 1 \mathrm{H}), 7.87(\mathrm{~s}, 1 \mathrm{H})$, $8.15(\mathrm{~s}, 1 \mathrm{H})$.

Compound 4: White solid, yield: $32 \%$, m.p. $147-148^{\circ} \mathrm{C}$; IR $\left(\mathrm{KBr}, v_{\max },\left(\mathrm{cm}^{-1}\right): 3325,3272,3130,3099,1737,1675\right.$, 1608, 1524, 1487, 1446, 1353, 1333, 1229, 1199, 1134, 814, 758, 737, 677, 590; ${ }^{1} \mathrm{H}$ NMR (400 MHz, $\left.\mathrm{CDCl}_{3}\right): \delta(\mathrm{ppm})$ : $2.35(\mathrm{~s}, 3 \mathrm{H}), 7.17(\mathrm{~d}, J=8.0 \mathrm{~Hz}, 1 \mathrm{H}), 7.35(\mathrm{t}, J=8.0 \mathrm{~Hz}, 1 \mathrm{H})$, $7.50-7.56(\mathrm{~m}, 2 \mathrm{H}), 7.80(\mathrm{t}, J=6.8 \mathrm{~Hz}, 2 \mathrm{H}), 7.81(\mathrm{~d}, J=8.0$ $\mathrm{Hz}, 1 \mathrm{H}), 8.34$ (s, 1H), 8.45 (s, 1H)

Compound 5: White solid, yield: $75 \%$, m.p. $95-96{ }^{\circ} \mathrm{C}$; IR $\left(\mathrm{KBr}, v_{\max }\left(\mathrm{cm}^{-1}\right): 3338,3200,3074,3000,2838,1651,1597\right.$, 1524, 1465, 1323, 1250, 1185, 1184, 1102, 1028, 753, 690, 595; ${ }^{1} \mathrm{H}$ NMR (400 MHz, $\mathrm{CDCl}_{3}$ ): $\delta(\mathrm{ppm}): 4.05$ (s, 3H), 7.03 $(\mathrm{d}, J=8.4 \mathrm{~Hz}, 1 \mathrm{H}), 7.11-7.15(\mathrm{~m}, 2 \mathrm{H}), 7.36(\mathrm{t}, J=8.0 \mathrm{~Hz}$, $2 \mathrm{H}), 7.47-7.51(\mathrm{~m}, 1 \mathrm{H}), 7.68(\mathrm{~d}, J=8.0 \mathrm{~Hz}, 2 \mathrm{H}), 8.28-8.30$ $(\mathrm{m}, 1 \mathrm{H}), 9.81(\mathrm{~m}, 1 \mathrm{H})$

Compound 6: Light yellow needle crystal, yield: $70 \%$, m.p. $97-98^{\circ} \mathrm{C}$; IR $\left(\mathrm{KBr}, v_{\max }, \mathrm{cm}^{-1}\right)$ : 3329, 3284, 3075, 3027, 2842, 1647, 1601, 1579, 1464, 1317, 1287, 1210, 1181, 1094, 1022, 831, 759, 638, 591; ${ }^{1} \mathrm{H}$ NMR (400 MHz, $\left.\mathrm{CDCl}_{3}\right): \delta$ (ppm), $4.05(\mathrm{~s}, 3 \mathrm{H}), 7.02-7.07(\mathrm{~m}, 3 \mathrm{H}), 7.13(\mathrm{t}, J=8.0 \mathrm{~Hz}$, $1 \mathrm{H}), 7.48-7.52(\mathrm{~m}, 1 \mathrm{H}), 7.62-7.65(\mathrm{~m}, 2 \mathrm{H}), 8.27-8.29(\mathrm{~m}, 1 \mathrm{H})$, $9.78(\mathrm{~s}, 1 \mathrm{H})$.

Compound 7: White needle crystal, yield: $80 \%$, m.p. 92-93 ${ }^{\circ} \mathrm{C}$; IR $\left(\mathrm{KBr}, v_{\max }, \mathrm{cm}^{-1}\right): 3345,3107,3031,2983,2843$, 1670, 1650, 1593, 1463, 1399, 1316, 1245, 1181, 1022, 1014, $825,759,689,619,590 ;{ }^{1} \mathrm{H} \mathrm{NMR}\left(400 \mathrm{MHz}, \mathrm{CDCl}_{3}\right): \delta(\mathrm{ppm})$ : $4.05(\mathrm{~s}, 3 \mathrm{H}), 7.03(\mathrm{~d}, J=8.4 \mathrm{~Hz}, 1 \mathrm{H}), 7.13(\mathrm{t}, J=8.0 \mathrm{~Hz}, 1 \mathrm{H})$, $7.30(\mathrm{t}, J=8.8 \mathrm{~Hz}, 2 \mathrm{H}), 7.48-7.52(\mathrm{~m}, 1 \mathrm{H}), 7.63(\mathrm{~d}, J=8.8$ $\mathrm{Hz}, 2 \mathrm{H}), 8.26-8.28(\mathrm{~m}, 1 \mathrm{H}), 9.83(\mathrm{~s}, 1 \mathrm{H})$.

Compound 8: White powder, yield: $65 \%$, m.p. 124$125^{\circ} \mathrm{C}$; IR (KBr, $\left.v_{\max }, \mathrm{cm}^{-1}\right): 3334,3080,3006,2932,2835$, $1655,1589,1518,1465,1316,1248,1163,1025,1013,819$, 757, 629, 618; ${ }^{1} \mathrm{H}$ NMR (400 MHz, $\left.\mathrm{CDCl}_{3}\right): \delta(\mathrm{ppm}): 4.05(\mathrm{~s}$, $3 \mathrm{H}), 7.03(\mathrm{~d}, J=8.4 \mathrm{~Hz}, 1 \mathrm{H}), 7.13(\mathrm{t}, J=7.2 \mathrm{~Hz}, 1 \mathrm{H}), 7.46(\mathrm{t}$, $J=6.8 \mathrm{~Hz}, 2 \mathrm{H}), 7.49-7.52(\mathrm{~m}, 1 \mathrm{H}), 7.58(\mathrm{~d}, J=8.8 \mathrm{~Hz}, 2 \mathrm{H})$, 8.26-8.28 (m, 1H), $9.83(\mathrm{~s}, 1 \mathrm{H})$.

Compound 9: Golden yellow crystal, yield: $58 \%$, m.p. $157-159^{\circ} \mathrm{C}$; IR $\left(\mathrm{KBr}, v_{\max }, \mathrm{cm}^{-1}\right): 3280,3256,3074,2987,2842$, $1668,1580,1464,1425,1343,1273,1148,1118,1045,1014$, 826, 745, 741, 686; ${ }^{1} \mathrm{H}$ NMR (400 MHz, $\mathrm{CDCl}_{3}$ ): $\delta$ (ppm): 4.14 $(\mathrm{s}, 3 \mathrm{H}), 7.06(\mathrm{~d}, J=8.0 \mathrm{~Hz}, 1 \mathrm{H}), 7.12$ (t, $J=8.0 \mathrm{~Hz}, 1 \mathrm{H}), 7.17$ $7.21(\mathrm{~m}, 1 \mathrm{H}), 7.51-7.56(\mathrm{~m}, 1 \mathrm{H}), 7.64-7.68(\mathrm{~m}, 1 \mathrm{H}), 8.19-8.21$ (m, 1H), 8.26-8.28 (m, 1H), 8.98-9.00 (m, 1H), $12.12(\mathrm{~s}, 1 \mathrm{H})$.
Compound 10: White crystal, yield: $83 \%$, m.p. $66-67{ }^{\circ} \mathrm{C}$; IR $\left(\mathrm{KBr}, v_{\max }\left(\mathrm{cm}^{-1}\right): 3348,3068,3015,2833,1660,1556\right.$, 1513, 1462, 1414, 1322, 1243, 1174, 1047, 1017, 833, 827, 753, 665, 600; ${ }^{1} \mathrm{H}$ NMR (400 MHz, $\left.\mathrm{CDCl}_{3}\right): \delta(\mathrm{ppm}): 3.80(\mathrm{~s}$, $3 \mathrm{H}), 4.02(\mathrm{~s}, 3 \mathrm{H}), 6.89(\mathrm{~d}, J=8.8 \mathrm{~Hz}, 2 \mathrm{H}), 7.01(\mathrm{~d}, J=8.4 \mathrm{~Hz}$, $1 \mathrm{H}), 7.12(\mathrm{t}, J=8.0 \mathrm{~Hz}, 1 \mathrm{H}), 7.45-7.49(\mathrm{~m}, 1 \mathrm{H}), 7.57-7.60$ (m, 2H), 8.27-8.29 (m, 1H), 9.69 (s, 1H).

Compound 11: Light purple solid, yield: $60 \%$, m.p. 77$78^{\circ} \mathrm{C}$; IR (KBr, $v_{\max }\left(\mathrm{cm}^{-1}\right): 3348,3068,2960,2833,1660,155$, 1513, 1462, 1243, 1174, 1047, 1017, 833, 827, 753, 665, 600; ${ }^{1} \mathrm{H}$ NMR (400 MHz, $\left.\mathrm{CDCl}_{3}\right): \delta(\mathrm{ppm}): 3.80(\mathrm{~s}, 3 \mathrm{H}), 4.02(\mathrm{~s}$, $3 \mathrm{H}), 6.89(\mathrm{~d}, J=8.8 \mathrm{~Hz}, 2 \mathrm{H}), 7.01(\mathrm{~d}, J=8.4 \mathrm{~Hz}, 1 \mathrm{H}), 7.12(\mathrm{t}$, $J=8.0 \mathrm{~Hz}, 1 \mathrm{H}), 7.45-7.49(\mathrm{~m}, 1 \mathrm{H}), 7.57-7.60$ (m, 2H), 8.27$8.29(\mathrm{~m}, 1 \mathrm{H}), 9.69(\mathrm{~s}, 1 \mathrm{H})$.

Compound 12: White needle crystal, yield: $88 \%$, m.p. 144-145 ${ }^{\circ} \mathrm{C}$; IR $\left(\mathrm{KBr}, v_{\max }\left(\mathrm{cm}^{-1}\right): 3351,3071,3032,2940\right.$, 1669, 1587, 1534, 1485, 1463, 1408, 1319, 1272, 1186, 1126, 1023, 823, 755, 654, 592; ${ }^{1} \mathrm{H}$ NMR (400 MHz, $\left.\mathrm{CDCl}_{3}\right): \delta$ (ppm): 2.59 (s, 3H), 4.08 (s, 3H), 7.05 (d, $J=8.8 \mathrm{~Hz}, 1 \mathrm{H})$, $7.15(\mathrm{t}, J=7.2 \mathrm{~Hz}, 1 \mathrm{H}), 7.50-7.55(\mathrm{~m}, 1 \mathrm{H}), 7.78(\mathrm{~d}, J=8.8 \mathrm{~Hz}$, $2 \mathrm{H}), 7.97$ (d, $J=8.8 \mathrm{~Hz}, 2 \mathrm{H}), 8.27-8.29$ (m, 1H), 10.05 (s, $1 \mathrm{H})$.

Compound 13: :Light yellow powder, yield: $85 \%$, m.p. 68-69 ${ }^{\circ} \mathrm{C}$; IR $\left(\mathrm{KBr}, v_{\max }\left(\mathrm{cm}^{-1}\right)\right.$ : 3447, 3392, 3072, 3029, 2847 , 1669, 1604, 1 492, 1457, 1326, 1243, 1146, 1072, 1016, 768, 746, 680; ${ }^{1} \mathrm{H}$ NMR (400 MHz, $\left.\mathrm{CDCl}_{3}\right): \delta(\mathrm{ppm}): 4.05$ (s, 3H), 6.79-6.84 (m, 1H), $7.03(\mathrm{~d}, J=8.4 \mathrm{~Hz}, 1 \mathrm{H}), 7.11-7.15(\mathrm{~m}$, $1 \mathrm{H}), 7.25-7.29(\mathrm{~m}, 2 \mathrm{H}), 7.48-7.52(\mathrm{~m}, 1 \mathrm{H}), 7.67-7.70(\mathrm{~m}, 1 \mathrm{H})$, 8.26-8.28 (m, 1H), 9.89 (s, 1H).

Compound 14: White needle crystal, yield: $87 \%$, m.p. 78-79 ${ }^{\circ} \mathrm{C}$; IR $\left(\mathrm{KBr}, v_{\max }, \mathrm{cm}^{-1}\right): 3446,3333,3082,3049,2839$, 1669, 1593, 1484, 1456, 1316, 1230, 1128, 1048, 1024, 791, 747, 684, 606; ${ }^{1} \mathrm{H}$ NMR (400 MHz, $\left.\mathrm{CDCl}_{3}\right): \delta$ (ppm): 4.06 (s, $3 \mathrm{H}), 7.03(\mathrm{~d}, J=8.4 \mathrm{~Hz}, 1 \mathrm{H}), 7.10(\mathrm{t}, J=6.0 \mathrm{~Hz}, 1 \mathrm{H}), 7.14(\mathrm{~d}$, $J=8.0 \mathrm{~Hz}, 1 \mathrm{H}), 7.48-7.53$ (m, 2H), 7.80 (s, 1H), 8.26-8.28 (m, 1H), 9.85 (s, 1H).

Compound 15: White needle crystal, yield: $85 \%$, m.p. 79-80 ${ }^{\circ} \mathrm{C}$; IR ( $\left.\mathrm{KBr}, v_{\max }, \mathrm{cm}^{-1}\right): 3438,3341,3069,2843,1665$, 1598, 1485, 1458, 1311, 1236, 1181, 1129, 1068, 1016, 779, 756, 681; ${ }^{1} \mathrm{H}$ NMR (400 MHz, $\left.\mathrm{CDCl}_{3}\right): \delta(\mathrm{ppm}): 4.06(\mathrm{~s}, 3 \mathrm{H})$, $7.03(\mathrm{~d}, J=8.4 \mathrm{~Hz}, 1 \mathrm{H}), 7.13(\mathrm{t}, J=8.0 \mathrm{~Hz}, 1 \mathrm{H}), 7.18-7.26$ $(\mathrm{m}, 2 \mathrm{H}), 7.48-7.53(\mathrm{~m}, 1 \mathrm{H}), 7.58-7.60(\mathrm{~m}, 1 \mathrm{H}), 7.92(\mathrm{~d}, J=$ $1.6 \mathrm{~Hz}, 1 \mathrm{H}), 8.25-8.28(\mathrm{~m}, 1 \mathrm{H}), 9.84(\mathrm{~s}, 1 \mathrm{H})$.

Compound 16: Light yellow crystal, yield: $80 \%$, m.p. 145-146 ${ }^{\circ} \mathrm{C}$; IR (KBr, $v_{\max }, \mathrm{cm}_{-1}$ ): 3312, 3203, 3123, 2986, 2843, 1669, 1552, 1531, 1486, 1467, 1340, 1258, 1236, 1130, 1073, 1020, 804, 750, 738, 676; ${ }^{1} \mathrm{H}$ NMR (400 MHz, $\left.\mathrm{CDCl}_{3}\right)$ : $\delta$ (ppm): $4.11(\mathrm{~s}, 3 \mathrm{H}), 7.07(\mathrm{~d}, J=8.4 \mathrm{~Hz}, 1 \mathrm{H}), 7.16(\mathrm{t}, J=8.0$ $\mathrm{Hz}, 1 \mathrm{H}), 7.49-7.56(\mathrm{~m}, 2 \mathrm{H}), 7.95-7.97(\mathrm{~m}, 1 \mathrm{H}), 8.27-8.30(\mathrm{~m}$, $1 \mathrm{H}), 8.49(\mathrm{t}, J=2.0 \mathrm{~Hz}, 1 \mathrm{H}), 10.07(\mathrm{~s}, 1 \mathrm{H})$.

Assay of antifungal activity: All the synthesized compounds were screened for their activity against Sclerotinia sclerotiorum and Bipolaris maydis(Nisikado et Miyake) Shoem using the plate growth rate method ${ }^{19}$.

Each tested compound and carbendazim (purity $90 \%$ ) were dissolved in dimethyl sulfoxide, respectively. They were added to the sterile culture medium (PDA) at $45^{\circ} \mathrm{C}$, mixed to homogeneity and transferred to sterile Petri dishes to solidify. 
For primary screening, each compound was used at a concentration of $100 \mathrm{mg} \mathrm{L}^{-1}$. A mycelium agar disc ( $5 \mathrm{~mm}$ in diameter) of the target fungi was placed in the center of PDA plates. They were incubated at $28^{\circ} \mathrm{C}$ in the dark until the target fungi used as the controls covered the surface of these plates. Control groups were treated with the corresponding solutions without the tested compound or carbendazim. The diameter of the fungi in the cultures was measured and the inhibition of growth was calculated according to Abbott's formula. Each compound was replicated three times.

\section{RESULTS AND DISCUSSION}

All the synthesized analogues of amide were screened for their activity against Sclerotinia sclerotiorum and Bipolaris maydis (Nisikado et Miyake) Shoem. The results are presented in Table-1. Some of them were active against the two different pathogenic fungi at a concentration of $100 \mathrm{mg} \mathrm{L}^{-1}$. Among them, the inhibition rate of compound $\mathbf{1}$ and compound $\mathbf{2}$ reached $91.1 \%, 92.8 \%$ and $90.1 \%, 90.1 \%$, respectively, which was close to carbendazim.

TABLE-1

ANTIFUNGAL ACTIVITY AGAINST Sclerotinia sclerotiorum AND Bipolaris maydis(Nisikado et Miyake) Shoem 1-16 AT $100 \mathrm{mg} \mathrm{L}^{-1}$

\begin{tabular}{cllcc}
\hline & & & \multicolumn{2}{c}{ Inhibition of growth (\%)* } \\
\cline { 3 - 5 } Comp. & $\mathrm{R} 1$ & $\mathrm{R} 2$ & $\begin{array}{c}\text { Sclerotinia } \\
\text { sclerotiorum }\end{array}$ & $\begin{array}{c}\text { Bipolaris maydis } \\
\text { (Nisikado et } \\
\text { Miyake) Shoem }\end{array}$ \\
\hline $\mathbf{1}$ & $3-\mathrm{F}$ & $\mathrm{OCOCH}_{3}$ & 91.1 & 90.1 \\
$\mathbf{2}$ & $3-\mathrm{Cl}$ & $\mathrm{OCOCH}_{3}$ & 92.8 & 90.1 \\
$\mathbf{3}$ & $4-\mathrm{Br}$ & $\mathrm{OCOCH}_{3}$ & 77.7 & 85.0 \\
$\mathbf{4}$ & $3-\mathrm{NO}_{2}$ & $\mathrm{OCOCH}_{3}$ & 92.8 & 74.1 \\
$\mathbf{5}$ & $\mathrm{H}$ & $\mathrm{OCH}_{3}$ & 88.8 & 50.9 \\
$\mathbf{6}$ & $4-\mathrm{F}$ & $\mathrm{OCH}_{3}$ & 62.0 & 87.6 \\
$\mathbf{7}$ & $4-\mathrm{Cl}$ & $\mathrm{OCH}_{3}$ & 23.4 & 63.5 \\
$\mathbf{8}$ & $4-\mathrm{Br}$ & $\mathrm{OCH}_{3}$ & -2.6 & 17.7 \\
$\mathbf{9}$ & $4-\mathrm{NO}_{2}$ & $\mathrm{OCH}_{3}$ & -0.3 & 9.7 \\
$\mathbf{1 0}$ & $4-\mathrm{CH}$ & $\mathrm{OCH}_{3}$ & 74.2 & 69.4 \\
$\mathbf{1 1}$ & $4-\mathrm{OCH}_{3}$ & $\mathrm{OCH}_{3}$ & 49.2 & 65.4 \\
$\mathbf{1 2}$ & $4-\mathrm{COCH}_{3}$ & $\mathrm{OCH}_{3}$ & 1.0 & 14.8 \\
$\mathbf{1 3}$ & $3-\mathrm{F}$ & $\mathrm{OCH}_{3}$ & 44.4 & 57.56 \\
$\mathbf{1 4}$ & $3-\mathrm{Cl}$ & $\mathrm{OCH}_{3}$ & 56.6 & 54.5 \\
$\mathbf{1 5}$ & $3-\mathrm{Br}$ & $\mathrm{OCH}_{3}$ & 65.1 & 37.1 \\
$\mathbf{1 6}$ & 3-NO & $\mathrm{OCH}_{3}$ & 11.97 & 13.46 \\
\hline \multicolumn{2}{l}{ Carbendazim } & & 99.8 & 91.0 \\
\hline Based on the mean of triplicates & &
\end{tabular}

A series of analogues of amide have been successfully synthesized and tested for their antifungal activity against Sclerotinia sclerotiorum and Bipolaris maydis (Nisikado et Miyake) Shoem. The preliminary results suggested that the design and synthesis of these compounds may be conducive to the antifungal activity of analogues of amide. They are also promising and beneficial to further studies in developing new and more effective fungicides in the agricultural chemistry field. However, there is more work to be done. A number of analogues of amide should be further synthesized for screening and surveying quantitative structure-activity relationships so as to find novel fungicides with high effect and low toxicity. The structural modification and optimization of compound $\mathbf{1}$ and compound $\mathbf{2}$ need to be done. Meanwhile, its mode of action and its safety to humans and non-target organisms also need to be investigated.

\section{ACKNOWLEDGEMENTS}

This project was supported by the Scientific Research Fund of Sichuan Provincial Education Department (No. 09ZA158 ) and the Key Scientific Research Fund of Xihua University (No. R0820501).

\section{REFERENCES}

1. M.J. Gilbert, C.R. Thornton, E. Gavin, G.E. Wakley and N.J. Talbot, Nature, 440, 535 (2006).

2. L.P. Partida-Martinez and C. Hertweck, Nature, 437, 884 (2005).

3. M.T. McGrath, Plant Dis., 85, 236 (2001).

4. J.Y. Cho, H.Y. Kim, G.J. Choi, K.S. Jang, H.K. Lim, C.H. Lim, K.Y. Cho and J.C. Kim, Pest Manage. Sci., 62, 414 (2006).

5. J.Y. Cho, G.J. Choi, S.W. Son, K.S. Jang, H.K. Lim, S.O. Lee, N.D. Sung, K.Y. Cho and J.C. Kim, Pest Manage. Sci., 63, 935 (2007).

6. M.A. Abbassy, S.A.M. Abdelgaleil, A.S.H. Belal and M.A.A.A. Rasoul, Indian Crop. Prod., 26, 345 (2007).

7. K. A1-Aghabary, Z.J. Zhu and Q.H. Shi, J. Plant Nut., 27, 2101 (2007).

8. R.K. Sairam, G.C. Srivastava and S. Agarwal, Biologia Plantarum, 49, 85 (2005).

9. J. Kovacik, B. Klejdus, J. Hedbavny and M. Backor, Ecotoxicology, 18, 544 (2009).

10. H. Karlidag, E. Yildirim and M. Turan, Sci. Agric., 66, 180 (2009).

11. M. Snyman and M.J. Cronje, J. Exp. Bot., 59, 2125 (2008).

12. H.T. Liu, Y.Y. Liu, Q.H. Pan, H.R. Yang, J.C. Zhan and W.D. Huang, J. Exp. Bot., 57, 3337 (2006).

13. S.M. Clarke, L.A.J. Mur, J.E. Wood and I.M. Scott, Plant J., 38, 38 (2004).

14. B. Singh and K. Usha, Plant Growth Regul., 39, 137 (2003).

15. M.A. E1-Tayeb, Plant Growth Regul., 45, 215 (2005).

16. H. Tachi, K. Fukuda-Yamada, T. Kojima, M. Shiraiwa and H. Takahara, Plant Physiol. Biochem., 47, 73 (2009).

17. N. Yalpani, A.J. Enyedi, J. Leon and I. Raskin, Planta, 193, 372 (1994).

18. K. Mahdavian, K.M. Kalantari, M. Ghorbanli and M. Torkzade, Biologia Plantarum, 52, 170 (2008).

19. W. Huang and G.-F. Yang, Bioorg. Med. Chem., 14, 8280 (2006). 